\title{
Correspondence
}

\section{Rectal examination in appendicitis}

Sir,

I read with interest the article on rectal examination in appendicitis, ' but feel that the conclusions are invalid and cannot go unchallenged.

The fact that $80+121$, that is 201 of 328 children examined $(61 \cdot 5 \%)$ experienced severe or minor discomfort casts serious doubt on the competence of the operators performing the rectal examinations. In my experience, I find that even consultant physicians and surgeons have no idea about how to perform a rectal examination on a child. When I see a crying child on the end of someone's finger, I blame the operator and not the child.

Successful rectal examinations can be performed on most children if the operator takes time and care. The most important part of the examination is never to insert the finger. The patient should always be asked to strain down on the finger, no matter how long this takes to achieve. During the examination the child is reassured and talked to in words he understands. In most instances no restraint is needed or necessary.

Sixty one of 103 examinations in acute appendicitis were positive. ${ }^{1}$ The position of the appendix is not stated. A retrocaecal appendix for instance, which would be expected in approximately a third of cases, may not give rise to rectal tenderness.

A correctly performed rectal examination must remain as a valuable and important examination in the assessment of acute abdominal pain, and I think the conclusions drawn in the report are invalid.

\section{Reference \\ ${ }^{1}$ Dickson AP, MacKinlay GA. Rectal examination and acute appendicitis. Arch Dis Child 1985;60:666-7.}

$$
\begin{array}{r}
\text { N V FreEMAN } \\
\text { Wessex Regional Centre for Paediatric Surgery, } \\
\text { Southampton, } \\
\text { Hants }
\end{array}
$$

\section{Drs Dickson and MacKinlay comment:}

We thank Mr Freeman for his comments on our paper ${ }^{1}$ but do not agree that they cast doubt on our conclusions.

We are naturally always careful with regard to our technique of rectal examination, but in our experience even the most cooperative child still suffers some discomfort from the procedure. Furthermore, many children have great difficulty in following instructions and relaxing completely, probably in view of the impending intrusion, no matter how much time and care is taken. Experience with adults confirms that the procedure performed even in patients who understand its requirement, produces con- siderable unpleasantness. It may be of interest that when our report was presented to meetings of paediatric surgeons before submission for publication, the general feeling was that the responses of our group of children to rectal examination were less severe than most.

We agree that retrocaecal appendicitis may not give rise to rectal tenderness, and this probably partly accounts for. only $60 \%$ of our rectal examinations being positive. We fail, however, to see the point of this comment in relation to the main conclusion of our report, which is that acute appendicitis may be diagnosed without rectal examination in the vast majority of children on the basis of history and general examination. As stated in the paper, we regard rectal examination to be mandatory in all children presenting with an acute abdomen who do not have a clear diagnosis of acute appendicitis on abdominal assessment.

\section{Ceftazidime in neonatal infections}

Sir,

Having used ceftazidime monotherapy for the blind treatment of neonatal sepsis for the past two years, ${ }^{12}$ we read the paper by Low et $a^{3}$ with interest. From our experience of treating more than 400 babies, 30 of whom had bacteraemia, we cannot agree with the authors' conclusions that ceftazidime has only a theoretical role for neonatal use, or that it cannot be recommended as monotherapy before the results of bacteriological cultures are known. The five cases reported where treatment was considered to have been unsuccessful and upon which Low et al based their conclusions, raise serious questions. The patient with fatal Escherichia coli meningitis who failed to respond to ceftazidime had not responded to previous treatment with gentamicin or chloramphenicol. As the authors point out, neonatal meningitis has a mortality of 35 to $50 \%$ no matter what antibiotic is used. In one of the two cases of group B streptococcal sepsis where ceftazidime was thought to have failed, treatment was delayed because of an earlier diagnosis of hyaline membrane disease. Many units would consider penicillin plus gentamicin more appropriate once infection with group B streptococci had been confirmed. It also seems unduly harsh to consider as a ceftazidime failure the baby with Enterobacter cloacael enterococcal bacteraemia who, having been previously treated unsuccessfully with penicillin and gentamicin, died with 24 hours of starting ceftazidime. Finally, the activity of ceftazidime against staphylococci is known to be unremarkable and initial treatment with this antibiotic could only have been expected to hold the case of Staphylococcus aureus bacteraemia until specific treatment could be introduced. High ceftazidime concentrations were reported in four of five samples of cerebrospinal fluid but no mention was made of contamination with blood, small 